\title{
Spuren pleistozäner Vereisung in den Altos de Cuchumatanes, Guatemala
}

\author{
Von Stefan Hastenrath, Madison, Wis. USA
}

\author{
Mit 8 Abbildungen
}

\begin{abstract}
$\mathrm{Zus}$ a m menfassung. Feldbeobachtungen in den Altos de Cuchumatanes von Westguatemala erstreckten sich auf die SE-NW gerichteten tektonischen Täler von Llanos de San Miguel - Llano Ventura und Todos Santos, und das benachbarte Hochland mit Gipfeln um $3800 \mathrm{~m}$.

Als unmittelbarer Nachweis einer früheren Vereisung wurde talauf und nordwestlich der Llanos de Man Miguel in 3 470-3600 m ein Komplex von vier Stirn- bzw. Seitenmoränen gefunden. Die Moränen enthalten Felsblöcke von $50-100 \mathrm{~cm}$ und haben eine typische Höhe um $20 \mathrm{~m}$. Eine Fortsetzung der Seitenmoränen biegt vom Haupttal nordwärts. Das Hochtal Llanos de San Miguel Llano Ventura scheint vor allem im Talabschnitt südöstlich eines Sattelpunktes im Längsprofil einen großen Eiskuchen enthalten zu haben, der wesentlich von der hohen Bergkette im SW aus genährt worden sein muß. Im Zusammenhang betrachtet ist es wahrscheinlich, daß einige abflußlose Wannen um $3700 \mathrm{~m}$ in dem südwestlich gelegenen Hochland sowie einige Talungen um $3500-3650 \mathrm{~m}$ in den Bergen südwestlich des Todos Santos-Tals auch Eis enthielten. Indessen herrschen Karstenerscheinungen vor, was eine zuverlässige Bestimmung gewisser möglicherweise glazigener Formen ausschließt; der Moränenkomplex bleibt damit als direkter Beleg. Die dazugehörige Gleichgewichtslinie des Eismassenhaushalts dürfte um $3650 \mathrm{~m}$ gelegen haben. Diese Höhenlage und die räumliche Abfolge der Moränen passen zu denen benachbarter Gebirgsgegenden der mesoamerikanischen Landbrücke.
\end{abstract}

A b s t r a c t. The area of field work in the Altos de Cuchumatanes of W Guatemala includes the SE-NW oriented tectonic valleys of Llanos de San Miguel - Llano Ventura and Todos Santos, as well as the neighbouring highlands with summits around $3800 \mathrm{~m}$.

As immediate evidence of glaciation, a complex of four terminal/lateral moraines was found up valley and NW of the Llanos de San Miguel at 3 470-3600 m. The moraines contain boulders of $50-100 \mathrm{~cm}$ and have a typical height of about $20 \mathrm{~m}$. An extension of the lateral moraine ridges bulges from the main valley northward. The valley Llanos de San Miguel - Llano Ventura appears to have contained a large body of ice, particularly in a portion to the SE of a col in the valley bottom; ice supply from the high mountain ridge to the SW must have been essential. In context it appears likely that some drainageless basins near $3700 \mathrm{~m}$ in the highlands to the SW and some valleys of $3500-3650 \mathrm{~m}$ to the SW of the Todos Santos valley also contained ice. However, karst phenomena are dominant in the area, thus precluding an unambiguous identification of certain potentially glacial forms, and leaving the moraine complex as the prima facie evidence. The associated ice equilibrium line must have been situated in the vicinity of $3650 \mathrm{~m}$. This elevation and the spatial sequence of moraines are considered consistent with evidence in contiguous mountain regions of Middle America.

\section{Einleitung}

An den gegenwärtig vergletscherten Bergen der südamerikanischen Anden, der mexikanischen Sierren und des nordamerikanischen Felsengebirges sind Zeugen vorzeitlicher Vereisung offenkundig und altbekannt. Die Gebirge der mittelamerikanischen Landbrücke dagegen bleiben durchweg unterhalb der rezenten Schneegrenze, und erst Mitte der fünfziger Jahre konnte WEYL (1956) Feldbefunde einer vermutlich würmzeitlichen Vereisung der Cordillera de Talamanca Costa Ricas erbringen. Für die Höhenlage der pleistozänen Schneegrenze schlug WEYL den damals überraschend niedrigen Wert von $3500 \mathrm{~m}$ vor, mit dem Vorbehalt möglicher nacheiszeitlicher tektonischer Vorgänge. Dieser Wert konnte bei Feldarbeiten im Chirripó-Gebiet im Dezember 1972 (HASTENRATH 1974) nur bestätigt werden. 
In Anbetracht von WEYL's Ergebnissen für Costa Rica und der aus verschiedenen Arbeiten (Klebelsberg 1948-49; White 1956) folgenden wesentlich größeren Höhenlage an den mexikanischen Vulkanen verdient die Frage einer pleistozänen Vereisung in den Gebirgen des nördlichen Mittelamerika besonderes Interesse. Unter diesem Gesichtspunkt wurden denn auch in den Jahren 1961-62 mehrere der hohen Vulkane in der Pazifischen Küstenkordillere Guatemalas bestiegen, darunter auch der mit $4220 \mathrm{~m}$ höchste Berg Zentralamerikas, der Tajumulco (Hastenrath 1963). Fossile Glazial- oder Periglazialerscheinungen waren indessen nicht auszumachen. Es versteht sich, daß diese Berge ihre heutige Höhe erst in jüngerer Zeit erreicht haben mögen, und daß mögliche pleistozäne Klimazeugen sehr wohl durch spätere vulkanische Vorgänge beseitigt worden sein können. Freilich gab es zu Anfang der sechziger Jahre noch keinerlei topographisches Kartenmaterial.

Im Verlauf des letzten Jahrzehnts sind in Guatemala Luftbildaufnahmen selbst entlegener Gebirgsgegenden durchgeführt worden, und es sind ausgezeichnete topographische Karten im Maßstab 1:50000 erstellt worden (Institute Geográfico Nacional 1966). Damit ist endlich eine angemessene Grundlage auch für geomorphologische Kartierungen geschaffen. Im Anschluß an Feldarbeiten im Chirripó-Massiv Costa Ricas (Hastenrath 1974) erschien eine erneute Vergleichsuntersuchung im nördlichen Mittelamerika wünschenswert. Von besonderem Interesse waren dabei naturgemäß die Altos de Cuchumatanes im westlichen Guatemala, die lediglich bis um $3800 \mathrm{~m}$ aufragen, bei denen es sich aber um ein vergleichsweise altes Gebirge handelt. Die Feldbeobachtungen wurden vom 27. bis 31. Dezember 1972 durchgeführt. Gelegentlich eines Besuches beim Instituto Geográfico Nacional de Guatemala war es möglich, eine unveröffentlichte Dissertation von Anderson (1969) über das Blatt San Sebastián Huehuetenango kurz einzusehen, die auch Glazialerscheinungen erwähnt.

Luftbilder und 1:50000 Karten erwiesen sich bei den Feldaufnahmen als besonders wertvoll. Auf diesen Wanderungen wurden fortlaufend Ablesungen an einem AneroidHöhenmesser gemacht, die an Punkte bekannter Höhe angeschlossen wurden. Damit ist eine für die vorliegenden $Z$ wecke hinreichende Meßgenauigkeit gewährleistet. Daneben wurde ein gewöhnlicher Marschkompaß benutzt.

\section{2. Übersicht über das Untersuchungsgebiet}

Abb. 1 ist eine Orientierungsskizze für den bei dieser Gelegenheit begangenen Teil der Altos de Cuchumatanes. Isohypsen sind den amtlichen 1:50000 Karten entnommen. Marschrouten während der Feldarbeiten sind eingetragen.

Im ganzen sind die Altos de Cuchumatanes (Weyl 1961; Instituto Geográfico Nacional 1972) als eine gehobene, von SW nach NE gekippte Scholle vorzustellen; diese Vorgänge waren im SW mit Brüchen, im NE vermutlich mit Flexuren verbunden. Das Untersuchungsgebiet ist von mehreren NW-SE orientierten Bruchlinien durchzogen, die teils als gewaltige Hochtäler und intramontane Becken in Erscheinung treten. Prominente Beispiele sind die Llanos de Chemal, das von Llanos de San Miguel nach Llano Ventura sich erstreckende Hochtal und das Tal von Todos Santos Cuchumatanes. Der NE-Teil des Untersuchungsgebietes bis hin zum Abfall zum Todos Santos-Tal ist im wesentlichen aus Kreidekalk aufgebaut, was sich in einer ausgeprägten Karstmorphologie äußert. In den tieferen Lagen dieses Tals streichen Konglomerate, Sandsteine und Kalke der jurassischen Todos Santos-Formation aus. Das Gebirgsmassiv im SW des Todos Santos-Tals besteht hauptsächlich aus permo-karbonischen Schichten, die Schieferton, Sandstein und Kalk enthalten. 


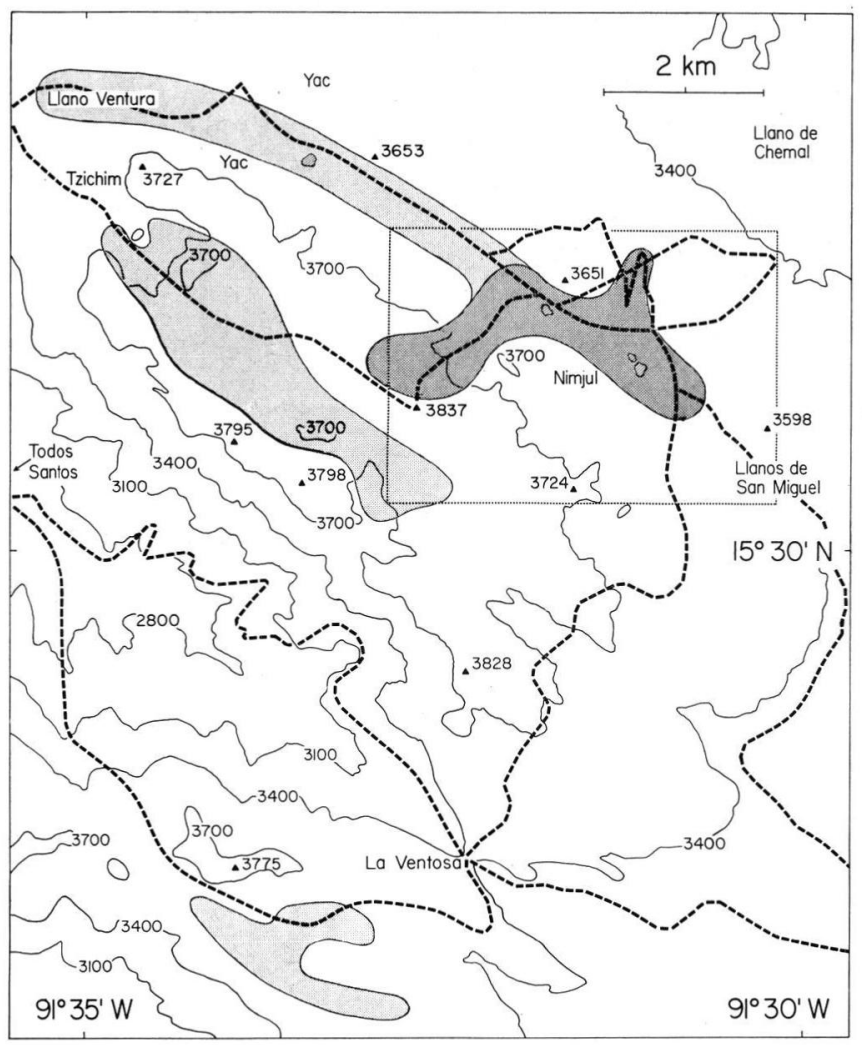

Abb. 1. Orientierungsskizze der Altos de Cuchumatanes. Höhenlinien sind den amtlichen $1: 50000$ Karten entnommen. Marschrouten sind als gestrichelte Linien eingetragen. Ehemals vereiste oder möglicherweise vereiste Flächen sind durch dunklere und hellere Schattierung gekennzeichnet; flache Tümpel sind schraffiert. Punktiertes Rechteck markiert das in Abb. 2 dargestellte Gebiet.

Dieses siedlungsfeindliche Hochland trägt auf den felsiger Bergrücken einen stattlichen Kiefernwald, der stellenweise stark mit Zypressen durchsetzt ist. Dazwischen erstrecken sich vor allem in den Hochtälern und intramontanen Becken ausgedehnte Graslandflächen. Die dürftige Landwirtschaft in den bescheidenen Siedlungen oberhalb etwa $3000 \mathrm{~m}$ beschränkt sich auf Schafzucht und etwas Kartoffelanbau.

Auf Grundlage der amtlichen Karten wurde ein Gebiet ausgesucht, für das die Möglichkeit einer pleistozänen Vereisung gemäß Höhenlage und Reliefverhältnissen am wahrscheinlichsten war. In den Abschnitten 3 bis 5 werden Beobachtungen in einzelnen Teilgebieten dieser Region besprochen.

\section{Hochtal zwischen Llanos de San Miguel und Llano Ventura}

Abb. 2 ist eine morphologische Skizze des SE-Abschnittes dieses Hochtals, und ein schematisches NW-SE Längsprofil ist in Abb. 3 wiedergegeben. Die Llanos de San Miguel, eine von bewaldeten Bergrücken umgebene offene Ebene um $3400 \mathrm{~m}$, bilden den SE-Eingang des Tales. Nach etwa $2 \mathrm{~km}$ steigt das Taltiefste stärker an, um nach 7,5 km einen Sattelpunkt um $3610 \mathrm{~m}$ zu erreichen. Jenseits dieses Sattels senkt sich der Talboden nach Llano Ventura zu auf $4,5 \mathrm{~km}$ um weniger als $100 \mathrm{~m}$ ab. Intermittierende Rinnsale und 
flache Tümpel kommen diesseits und jenseits des Sattelpunktes vor, der dabei eine Wasserscheide darstellt. Die weitere Fortsetzung des Tales südlich und westlich von Llano Ventura ist ungemein steil. Der geschlossene und bewaldete Bergkamm im SW des Tales Llanos de San Miguel - Llano Ventura steigt auf $2 \mathrm{~km}$ Horizontaldistanz auf um $3800 \mathrm{~m}$ an; die höchsten Berge fallen nach dem längeren südöstlichen Talabschnitt zu ab. Der meistenteils bewaldete Bergkamm im NE liegt näher zur Talachse, erreicht nur stellenweise um $3650 \mathrm{~m}$, und ist von niedrigeren Pässen unterbrochen.

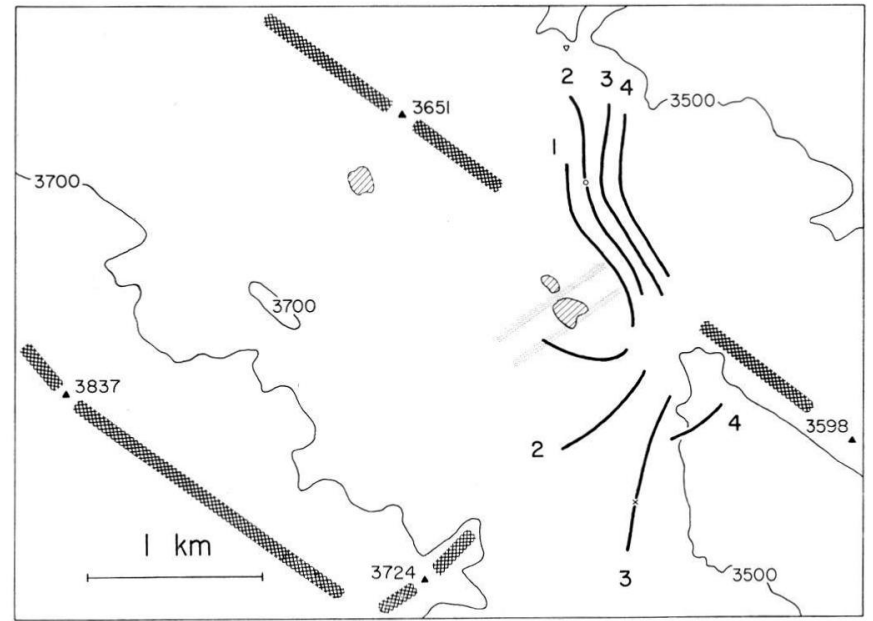

Abb. 2. Morphologische Skizze für den SE-Abschnitt des Hochtals Llanos de San Miguel - Llano Ventura. Höhenlinien sind den amtlichen 1:50000 Karten entnommen. Felsschwellen und Strukturlinien sind schattiert, Tümpel schraffiert, Bergkämme durch Kreuzschraffur gekennzeichnet, und Moränen kräftig ausgezogen. Standpunkte der Fotos Abb. 4, 5 und 6 sind durch Kreuz, Kreis bzw. Dreieck angezeigt.

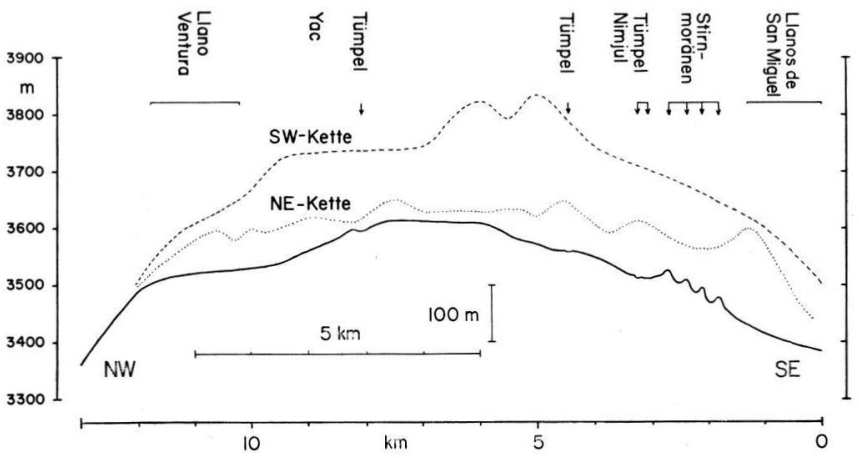

Abb. 3. Schematisches Längsprofil (NW-SE) des Hochtals Llanos de San Miguel-Llano Ventura. Überhöhung $1: 10$. Silhouette der Bergkette im SW gestrichelte Linie, Bergkette im NE punktiert.

Im Anstieg von den Llanos de San Miguel nordwestwärts, auf den oberen Teil des Tales zu, findet sich zwischen 3470 und $3600 \mathrm{~m}$ ein Komplex von vier großen Moränenkränzen. Diese seien im folgenden von NW nach SE fortschreitend als Moränen 1, 2, 3 und 4 bezeichnet (Abb.4). Die Moränen bestehen aus grobem Schuttmaterial mit Blöcken von $50-100 \mathrm{~cm}$ Durchmesser und erreichen Kammhöhen von um $20 \mathrm{~m}$. Von der Stirnpartie der untersten Moräne (4) sind nur Reste erhalten. Die oberen drei Moränen (1, 2, 3) 
ziehen von der südwestlichen Talflanke in konvex nach SE geschwungenen Bögen zur nordöstlichen Talseite, wo sie sich in Seitenmoränenwällen fortsetzen. Ein dazu paralleler, vierter Rücken ist deutlich auszumachen und ist wohl der untersten Endmoräne (4) zuzuschreiben. Lediglich die oberste Moräne (1) zeigt den Ansatz einer Seitenmoränenpartie auf der südwestlichen Talseite. Die zweitunterste Moräne (3) läßt in den oberen Partien auf der südwestlichen Talflanke ein Nebenstadium talab erkennen. Die zweitoberste Moräne (2) erscheint in ihrer Stirnpartie leicht aufgefächert. Teile der Moränenstränge sind weitständig mit Kiefern bestanden. Die oberste Moräne (1) bildet die Stirnwand eines sich talaufwärts anschließenden Kessels, der zwei seichte Tümpel enthält. Die Tümpel sind talab jeweils durch eine Felsschwelle begrenzt, deren Fortsetzung auf beiden Talseiten zutage tritt.

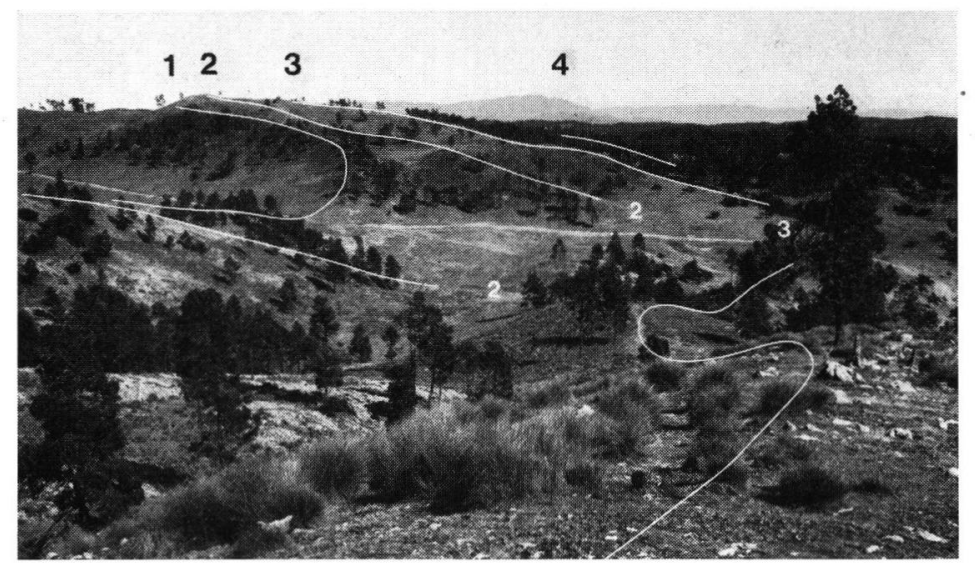

Abb. 4. Standpunkt $3600 \mathrm{~m}$ auf zweitunterster Seitenmoräne (3) auf SW-Seite des Tals Llanos de San Miguel-Llano Ventura, in Abb. 2 durch Kreuz markiert; Blick nach N auf die vier Moränenkränze. Kämme sind durch weiße Linien angedeutet und durch Zahlen gekennzeichnet.

Die vier Seitenmoränenrücken lassen sich zunächst ungefähr $1 \mathrm{~km}$ das Haupttal aufwärts nach NW verfolgen, wobei die zweitoberste Moräne (2) bei weitem die stattlichste ist. In einer Unterbrechung des parallel zum Haupttal SE-NW streichenden Felsrückens aber biegen die Moränenwälle nach $\mathrm{N}$ ab (Abb. 5). In einer nach ungefähr $\mathrm{N}$ sich absenkenden Talung laufen sie dann in Andeutungen von Stirnpartien aus. Die Moränenmorphologie wird ahnungslos von ein paar in cieser Talung hausenden Indianern ausgenützt: eine wohl in Zusammenhang mit der Schafhaltung angelegte Umzäunung verläuft entlang der zweitobersten Moräne (2), und ein in der Rinne zwischen zweitoberster (2) und zweitunterster (3) Moräne gelegener Tümpel muß verlockend genug gewesen sein, gerade dort eine Hütte zu errichten (Abb. 6).

Das Haupttal weiter nordwestwärts auf den Sattelpunkt zu folgend waren keine Anzeichen von Seiten- oder Stirnmoränen auszumachen. Der leicht gewellte Talboden, schliffkehlenartige Versteilung des am Talrand hervortretenden Gesteins, sowie die allenthalben anzutreffenden glatten Felsflächen und rundlichen Vertiefungen sind sicher im wesentlichen dem Karstformenschatz zuzuzählen. An den SW-Flanken des Tales konnten bis hinauf zum Kammniveau gleichfalls keinerlei Kar- oder Nivationsnischen ausgemacht werden, vielmehr war der Karstcharakter durchweg dominierend. Gletscherschrammen waren nirgends erhalten. 


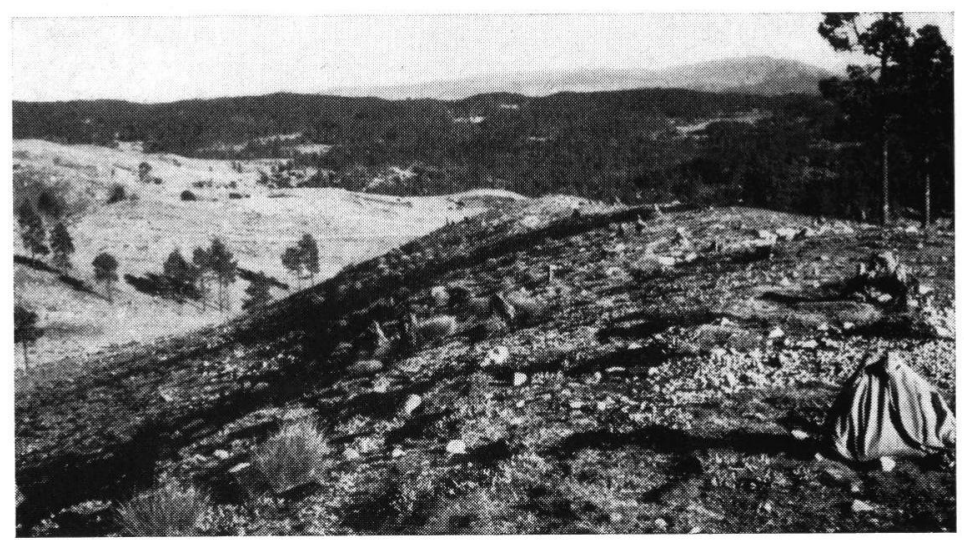

Abb. 5. Blick nach N entlang N-Ast der zweitobersten Seitenmoräne (2); $3580 \mathrm{~m}$. Standpunkt in Abb. 2 durch Kreis markiert.

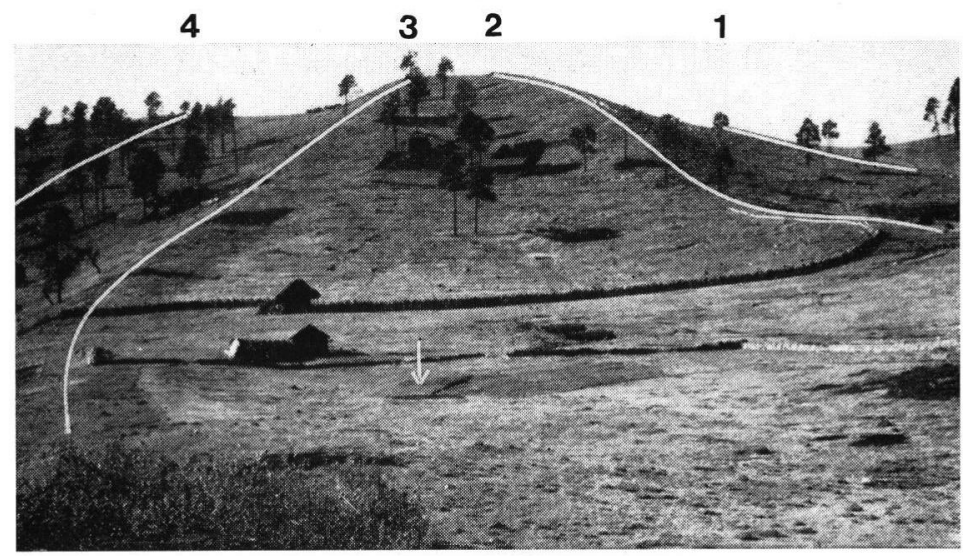

Abb. 6. Standpunkt $3500 \mathrm{~m}, 1,5 \mathrm{~km}$ nördlich des Haupttals, in Abb. 2 durch offenes Dreieck markiert; Blick nach SSW auf Ausläufer der Moränenrücken. Kammlinien sind durch weiße Linien angedeutet und durch Zahlen gekennzeichnet; Pfeil zeigt einen Tümpel an.

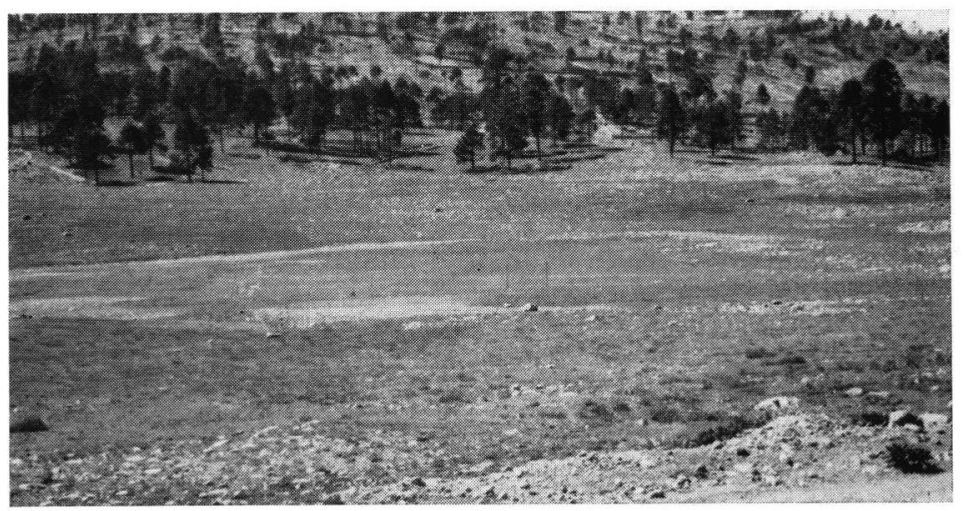

Abb. 7. Standpunkt nordwestlich des Sattelpunktes im Tal Llanos de San Miguel-Llano Ventura, $3600 \mathrm{~m}$. Blick nach WSW auf Talverebnung mit intermittierendem Tümpel. 
Gut $500 \mathrm{~m}$ jenseits des Sattelpunktes trifft man auf eine Verebnung (Abb. 7), die den im SE des Tals oberhalb des Moränenkomplexes gelegenen Tümpeln ähnlich ist. Während der Trockenzeit dient sie der Jugend von Yak als Fußballplatz, in der Regenzeit verwandelt sie sich in einen See. Auch sie ist anscheinend talab durch eine Felsschwelle begrenzt. Weiter talab, nach NW zu, dehnen sich im Talboden glatte und nahezu horizontale Felsflächen aus, die jedenfalls gelegentlich diskordant zu der Gesteinsschichtung zu sein scheinen. Schleffkehlenartige Versteilung des Talrandes ist auch hier ausgeprägt. Im Gegensatz zu den eindrucksvollen Moränenkränzen am SW-Ende des Tals, waren zwischen Sattelpunkt und Llano Ventura keine vergleichbaren Ablagerungen zu erkennen. Auch in der steilen Fortsetzung des Tals nach SW, S und NW fehlen offensichtlich Moränen.

\section{Hochland nordöstlich des Todos Santos-Tals}

Das Hochland zwischen dem Tal Llanos de San Miguel - Llano Ventura und dem Todos Santos-Tal enthält mehrere von bewaldeten Felsrücken umrahmte flache Wannen um $3700 \mathrm{~m}$, deren Achse SE-NW ausgerichtet ist. Die Wannen mit typischer Ausdehnung von $1-2 \mathrm{~km}$ sind ohne oberflächlichen Abfluß und tragen offenes Grasland. Am Boden der Wannen kommen nahezu ebene Felsflächen zum Vorschein, die von Karren zerschnitten sind (Abb. 8). An den Wannenrändern treten schliffkehlenartige Versteilungen des anstehenden Kalkgesteins auf, und zwar besonders auffallend in zwei weiten Mulden, die von Wannen am Westrand dieses Hochlandes zur Talsiedlung von Tzichim (3450_ $3550 \mathrm{~m}$ ) hinunterleiten (Abb. 1).

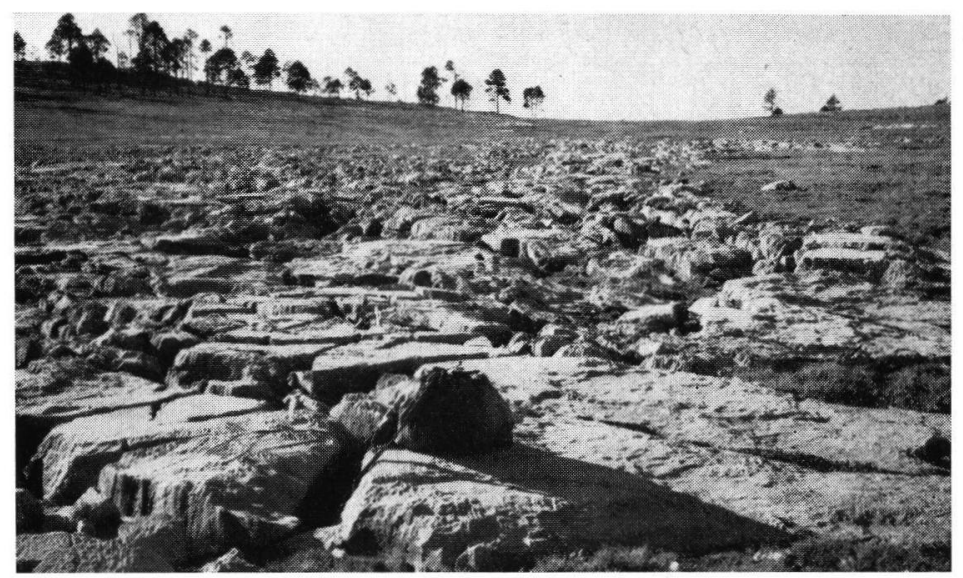

Abb. 8. Auf Hochland zwischen Tal Llanos de San Miguel-Llano Ventura und Todos Santos-Tal, $3700 \mathrm{~m}$. Blick nach WNW; von Karren zerschnittene ebene Felsfläche, im Hintergrund Rand der Wanne.

\section{Tal von Todos Santos und das im SW anschließende Hochland}

Das Todos Santos-Tal (Abb. 1) zeigt ein ähnliches Längsprofil wie das Tal Llanos de San Miguel-Llano Ventura, nur daß vom Sattelpunkt (La Ventosa) der Talboden ungleich steiler nach NW und SE abfällt. Die Sattelpunkte am Boden der beiden Täler und die höchsten Berggipfel des dazwischenliegenden Hochlandes liegen auf einer nicht ganz $\mathrm{N}-\mathrm{S}$ verlaufenden Linie. Die ungewöhnliche Reliefenergie des Todos Santos-Tals ist offenbar tektonisch bedingt. Im Gelände waren auch mit gleichzeitiger Ausnutzung von 
Luftbildern und topographischer Karte weder Moränen noch andere glazigene Formbildungen zu erkennen.

In dem an das Todos Santos-Tal nach SW anschließenden Hochland (Abb. 1) gibt es weite muldenförmige Becken und Talungen um $3500-3650 \mathrm{~m}$, die im Gegensatz zu den umrahmenden Falskämmen offenes Grasland tragen und in den Großformen an Nivationsbecken erinnern.

\section{Periglazialerscheinungen}

An fossilen Periglazialbildungen wurde im Tal Llanos de San Miguel-Llano Ventura etwa $1,5 \mathrm{~km}$ nordwestlich des obersten Stirnmoränenbogens (1) in einem Aufschluß um $3570 \mathrm{~m}$ eine Solifluktionsschuttdecke beobachtet.

An rezenten Formbildungen finden sich oberhalb $3500 \mathrm{~m}$ allenthalben Mikrovegetationsterrassen und Pipkrake, deren Feinerdereste eine ungefähr W-E orientierte Streifenanordnung zeigen, die anscheinend dem lokalen Sonnenaufgang entspricht.

\section{Schlußfolgerungen}

Die mächtigen Stirn- und Seitenmoränen nordwestlich der Llanos de San Miguel sind eindeutige Zeugen pleistozäner Vereisung; die Frischheit der Formen deutet auf ein dem Würm entsprechendes Alter. Beachtenswert ist das Fehlen auch nur annähernd vergleichbarer Moränen am NW-Ausgang des Tals. Es ist einmal zu überlegen, daß mögliche Moränenablagerungen in der steilen Talfortsetzung jenseits der Llanos Ventura leicht verloren gegangen sein konnten. Noch wichtiger ist der Umstand, daß der Sattelpunkt das Tal in einen recht langen SE- und einen viel kürzeren NW-Abschnitt teilt. Das dürfte die Eiszufuhr in den Raum des Llano Ventura benachteiligt haben, zumal die höchsten Rükken der Bergkette im SW zum Einzugsbereich des südöstlichen Talabschnitts gehören; es sind die höchsten Gipfel der Altos de Cuchumatanes überhaupt. Es will scheinen, daß nirgends sonst in den Altos de Cuchumatanes die Bedingungen zur Eisansammlung so günstig gewesen sein können wie in dem SE-Abschnitt des Tales Llanos de San MiguelLlano Ventura. Vor allem dieser Talabschnitt dürfte von einem großen Eiskuchen ausgefüllt gewesen sein, wobei ein Eislobus vom Haupttal aus durch einen $\mathrm{Pa} ß$ in dem im $\mathrm{NE}$ talparallel verlaufenden Felsriegel nach $\mathrm{N}$ vordrang. Der Eiskuchen im Haupttal mochte wesentlich durch Zufuhr von der hohen Bergkette im SW genährt worden sein.

In Anbetracht der überzeugenden Moränenkränze nordwestlich der Llanos de San Miguel liegt es nahe, auf dem noch wesentlich höheren Hochplateau im SW erst recht eine Vereisung zu erwarten. Der in Abschnitt 4 skizzierte Formenschatz könnte demnach dahingehend gedeutet werden, daß die ausgedehnten Wannen Eisfelder enthielten, aus denen die heute bewaldeten Felsrücken hervorragten; im W des Plateaus mochte das Eis zum Tal von Tzichim abgeflossen sein. Sollte eine derartige Interpretation lediglich aus dem Zusammenhang heraus berechtigt erscheinen, so ist zu betonen, daß eindeutige Moränenwälle und Gletscherschrammen in diesem Gebiet nicht aufzuspüren waren, womit jedenfalls ein unmittelbarer Nachweis entfällt.

Es ist vorstellbar, daß einige der in Abschnitt 5 erwähnten Talungen im Hochland südwestlich des Todos Santos-Tals einst von Eis erfüllt gewesen sind, aber ein unmittelbarer Beleg etwa auf Grund von Moränenrücken und Gletscherschrammen war auch hier nicht zu erbringen.

Der Nachweis einwandfrei glazialer Formen wird durch die ausgeprägte Karstmorphologie des Gebietes erschwert. Hohlformen mit vorgelagerter Felsschwelle sind nicht einer Enstehung als Kare oder Nivationsnischen zuzuschreiben, denn man findet Dolinen 
allenthalben im Gelände. Glatte Felsflächen, auch diskordant mit der Schichtung können durch chemische Verwitterung zustande kommen und verdanken ihre Entstehung nicht unbedingt glazialer Überformung. Es besteht keine Hoffnung, daß auf diesem Gestein Gletscherschrammen erhalten bleiben. Bei der Interpretation von Schuttdecken ist ganz allgemein Vorsicht am Platze. Lediglich für Moränenwälle in klarer räumlicher Anordnung läßt sich der glaziale Ursprung ausmachen.

Die Möglichkeit von Eisfeldern um $3700 \mathrm{~m}$ auf dem von den beiden großen tektonischen Tälern begrenzten Hochplateau sowie in einigen Talungen um $3500-3650 \mathrm{~m}$ in den Bergen südwestlich des Todos Santos-Tals gewinnt lediglich im Zusammenhang Wahrscheinlichkeit. Als unmittelbarer Nachweis bleibt damit der große Moränenkomplex nordwestlich der Llanos de San Miguel. Für die dazugehörige Gleichgewichtslinie des Eismassenhaushalts sei eine Höhenlage um $3650 \mathrm{~m}$ vorgeschlagen. Daß diese Linie sehr viel unterhalb 3600 m gelegen haben könnte, ist den Reliefverhältnissen nach wenig wahrscheinlich. Andererseits wäre es bei einer Höhenlage wesentlich über $3700 \mathrm{~m}$ schwer vorstellbar, daß die gewaltigen Moränensysteme weit talab hätten aufgebaut werden können.

Abschließend seien die Feldbeobachtungen mit Befunden in benachbarten Gebirgen der amerikanischen Tropen verglichen. In den Anden von Venezuela konnte SchuberT (1970) fünf Hauptmoränenzüge nachweisen. In den Kolumbischen Anden entdeckten ConzÁLez et al. (1965) vier ausgeprägte Moränenkomplexe, von denen aber der jüngste für nicht älter als 300 Jahre gehalten wird. Bei kürzlichen Geländearbeiten im Chirripó-Gebiet der Cordillera de Talamanca Costa Ricas (Hastenrath 1974) konnten zumindest drei große Moränenzüge an einem Talausgang ausgemacht werden; weitere zumindest drei viel kleinere Moränen sind oberhalb davon im Talbecken eingebettet. Die pleistozäne Schneegrenzhöhe in der Cordillera de Talamanca ist mit WeYL (1956) um $3500 \mathrm{~m}$ anzusetzen, in guter räumlicher Übereinstimmung mit OpPenheim's (1940) ähnlichen Werten für die Kolumbischen Anden. Von der Breitenlage unseres Untersuchungsgebietes nach $\mathrm{N}$ fortschreitend, stehen die Arbeiten White's (1956) für die Vulkane der mexikanischen Meseta zur Verfügung. An der Iztaccíhuatl stellte White zumindest drei Glazialstadien in niederen Lagen, ein unbedeutendes Stadium in mittlerer Lage, und ein Stadium in größerer Höhe fest; außerdem finden sich drei bis vier kleine Moränenrücken in den Talschlüssen. Die pleistozäne Schneegrenze muß verschiedenen Arbeiten zufolge (KLEBELSBERG 1948-49; White 1956) höher gelegen haben als in Costa Rica, aber jedenfalls unterhalb des $4000 \mathrm{~m}$ Niveaus. An der Malinche im Raum von Puebla erkannten Heine und Heide-Weise (1972) drei Moränenphasen unterhalb der Waldgrenze um $3900 \mathrm{~m}$. Absolute Datierung ergab mehr als 21000 Jahre bis mehr als 8000 Jahre, während Moränen oberhalb der Baumgrenze der „kleinen Eiszeit“ zugeschrieben werden. Die Moränen werden mit White's Moränenbefunden an der Iztaccíhuatl in Beziehung gesetzt. Für den Südteil des nordamerikanischen Felsengebirges schließlich stellte RAY (1940) fünf Moränenphasen der Wisconsin-Vereisung fest, von denen die mittleren drei anscheinend den besten räumlichen Zusammenhang zeigen.

Wenngleich absolute Datierung und regionale Koordinierung der Moränen im zentralamerikanisch-mexikanischen Raum zumeist noch ausstehen, so ergänzen die vorgelegten Feldbeobachtungen in den Altos de Cuchumatanes mit Hinblick auf den meridionalen Verlauf der pleistozänen Schneegrenze und die räumliche Abfolge hauptsächlicher Moränenkomplexe sinnvoll Befunde in den benachbarten Gebieten der Landbrücke.

\section{D a n k :}

Prof. R. WeYl hat freundlicherweise ein Manuskript dieses Aufsatzes gelesen. Dr. S. Bonis war mir beim Besuch des Instituto Geográfico Nacional de Guatemala behilflich.

3 Eiszeitalter u. Gegenwart 


\section{Literaturverzeichnis}

Anderson, T. H. (1969): Geology of the San Sebastian Huehuetenango quadrangle, Guatemala. - Ph. D. Diss. Univ. Texas, Austin.

González, E., VAN DER Hammen, Th. \& Flint, R. F. (1965): Late quaternary glacial and vegetational sequence in Valle de Lagunillas, Sierra Nevada del Cocuy, Colombia. - Leidse Geol. Med., 32, 157-182.

HastenRath, S. (1963): Zum Einfluß der Massenerhebung auf den Verlauf der Klima- und Vegetationsstufen in Mittelamerika und im südlichen Mexiko. - Geografiska Annaler, 45, 76-83.

- (1974): On the pleistocene glaciation of the Cordillera de Talamanca, Costa Rica. - Z. f. Gletscherkunde u. Glazialgeologie, im Druck.

Heine, K. \& H. Heide-Weise (1972): Estratigrafía del pleistoceno reciente y del holoceno en el Volcán de la Malinche y región circunvecina. - Proyecto Puebla, Tlaxcala, Comunicaciones 5/1972, p. 3-7, Fundación Alemana para la Investigación Científica, Puebla, México.

Instituto Geográfico Nacional, 1966: Guatelama 1:50000; Blätter 1862 I und II.

Instituto Geográfico Nacional, 1970: Mapa geológico de Guatemala, $1: 500,000$.

Instituto Geográfico Nacional, 1972: Mapa geológico de Guatemala, 1:50000; Blatt 1862 II G.

Oppenherm, V. (1940): Glaciaciones cuaternarias en la Cordillera Oriental de Colombia. Revista Academia Colombiana de Ciencias Exactas, 4, no. 13, 70-81.

Ray, L. L. (1940): Glacial chronology of the southern Rocky Mountains. - Bull. Geol. Soc. America, 51, 1851-1918, 12 Abb., 4 Tab., Washington.

Schubert, C. (1970): Glaciation of the Sierra de Santo Domingo, Venezuelan Andes. - Quaternaria, 13, 225-246.

Klebelsberg, R. von (1948-49): Handbuch der Gletscherkunde und Glazialgeologie. - Wien, (Springer Verlag), 2 Bde., $1028 \mathrm{~S}$.

WEYL, R. (1956): Eiszeitliche Gletscherspuren in Costa Rica (Mittelamerika). - Z. f. Gletscherkunde u. Glazialgeologie, 3, 317-325, 2 Abb., 2 Taf., Innsbruck.

- (1961): Die Geologie Mittelamerikas. - 226 S. Berlin (Borntraeger).

White, S. E. (1956): Probable substages of glaciation on Iztaccíhuatl, Mexico. - J. Geol., 64, 289-295, 2 Abb., 1 Tab., Chicago.

Manuskript eingeg. 26. 3. 1973.

Anschrift des Verf.: Prof. Dr. S. Hastenrath, The University of Wisconsin, Dept. of Meteorology, 1225 West Dayton Street, Madison, Wis. 53706. 